\title{
15. The Mode of Inward Spread of Activation in Frog Muscle Fibres Investigated by Means of High-speed Microcinematography*)
}

\author{
By Haruo SUgI \\ Department of Physiology, Faculty of Medicine, University of Tokyo \\ (Comm. by Yasuji Katsuki, M. J. A., Jan. 12, 1973)
}

Although Huxley and Taylor ${ }^{4)}$ suggested that the influence of membrane depolarization in striated muscle fibres is conducted electrotonically inwards along the transverse tubular system ( $\mathrm{T}$ system), evidence has been accumulating that some degree of regenerating mechanism may be involved in the inward spread of activation, ${ }^{1)-3), 5)-7)}$ Sugi and $\mathrm{Ochi}^{\mathrm{T})}$ reported that local activation of frog fast muscle fibres with moderate depolarization produced graded contractions, while strong current initiated contractions spreading around the whole perimeter of the fibres. According to Strickholm ${ }^{5)}$ a complicated seasonal variation in both electrical and mechanical responses occurs between winter and summer frog fibres. Since the work of Sugi and Ochi was made only on winter frogs, the present experiments were undertaken to see whether local activation of summer frog fibres with moderate depolarization may produce any type of local contraction other than the graded one described by Huxley and Taylor.

Single fast muscle fibres showing all-or-none twitch responses were isolated from the semitendinosus muscles of the frog (Rana nigromaculata), and mounted horizontally in a glass trough for microscopic observation (Leitz UMK 5OX objective, N.A. 0.6). A limited area of surface membrane was depolarized by applying rectangular current pulses to a glass pipette whose tip (external diameter, $20-90 \mu$ ) was in contact with the fibre surface. The magnitude of local membrane depolarization was calculated by multiplying the contact resistance, i.e. the increase of resistance of the pipette when its tip was brought into contact with the fibre surface, by the applied current. ${ }^{6)}$ Local contractions were recorded with a $16 \mathrm{~mm}$ high-speed camera (Hycam) at 1,000-3,000 frames/sec, and contraction curves at a given part from the fibre surface were constructed by measuring the length of 5-10 sarcomeres with a film motion analyser (Vanguard). The experiments were performed from June to September, 1972 at room temperature $\left(20-24^{\circ} \mathrm{C}\right)$.

\footnotetext{
*) This paper is dedicated to Professor Haruo Kinosita on the occasion of his sixtieth birthday.
} 


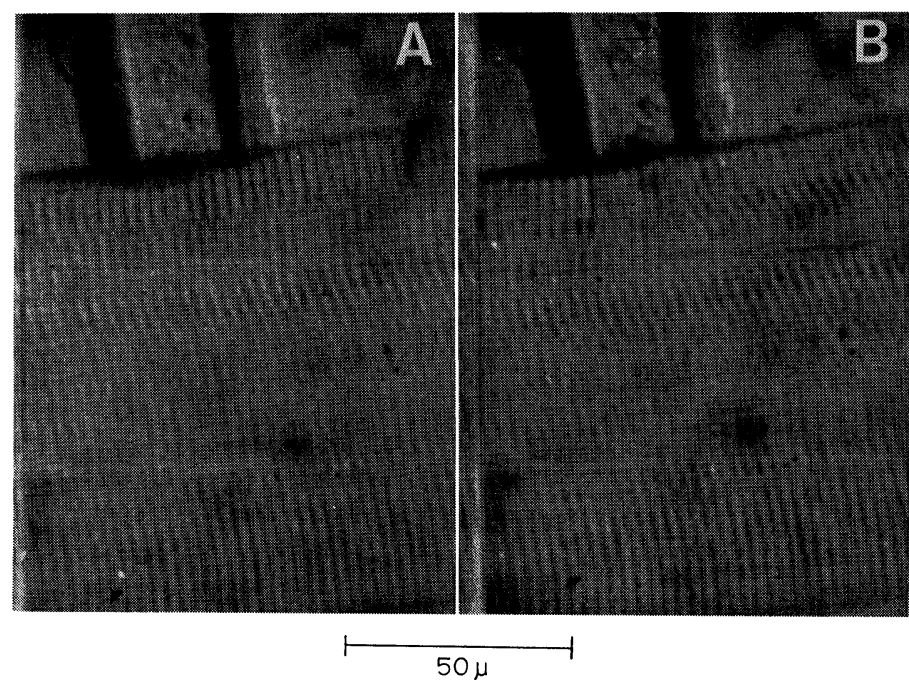

Fig. 1. Frames from a cinefilm of a resting muscle fibre (A) and of the local contraction (B) elicited by a $500 \mathrm{msec}$ current pulse producing depolarization of $25 \mathrm{mV}$. The pipette for local activation is in contact with the upper edge of the fibre.

In about $30 \%$ of the fibres studied, small depolarizations of 20 $30 \mathrm{mV}$ could elicit phasic local contractions which were found to relax spontaneously while current pulses producing depolarization still continued (Figs. 2 and 4), in contrast with the graded contractions which were normally initiated with depolarizations of above $40-50 \mathrm{mV}$, lasting as long as the current pulse went on.4),7) With pipettes of $20-40 \mu$ diameter, the contraction was first initiated at the fibre surface under the pipette, and spread to some extent inwards (Figs. 1 and 2). The phasic contraction at the fibre surface was followed by a small steady contraction which relaxed only after the current pulse was removed (Fig. 2A), whereas the contraction at the interior of the fibre relaxed completely within 100 msec (Fig. 2B).

Meanwhile, local activation of the fibres with pipettes of $50-90 \mu$ diameter revealed conspicuous features of the phasic response. As shown in Figs. 3 and 4, the contraction was first initiated at the interior of the fibre, and was observed to spread to the fibre surface. The contraction at the interior of the fibre occurred most markedly, and was followed by the small steady contraction lasting as long as the current pulse continued (Fig. 4B), while the contraction at the fibre surface relaxed within about $100 \mathrm{msec}$ (Fig. 4A). If the magnitude of depolarization was increased from 20 to $30 \mathrm{mV}$, the phasic contractions at both the fibre surface and interior were followed by the steady contractions. 


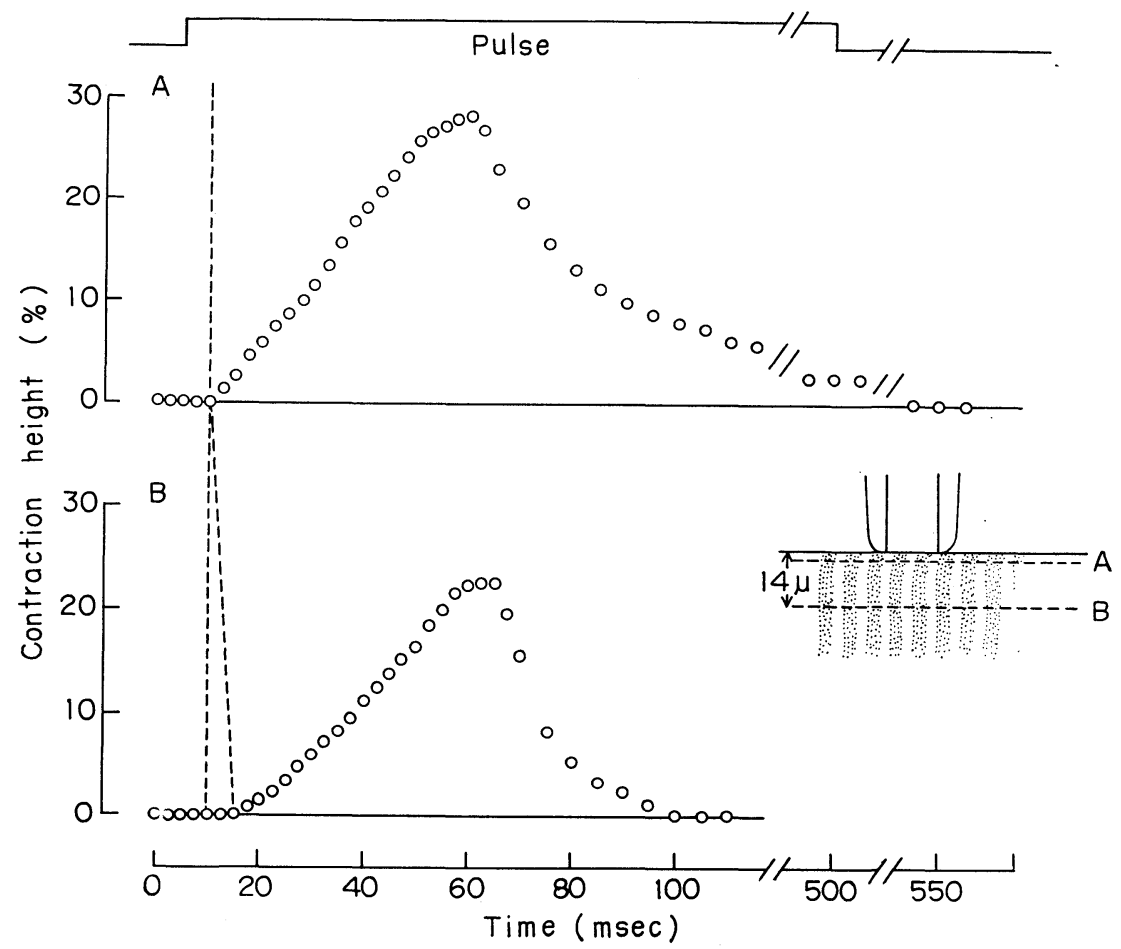

Fig. 2. Contraction curves of the local contraction shown in Fig. 1 constructed by measuling the length of 5 sarcomeres near the fibre surface under the pipette (A) and at the part about $14 \mu$ distant from the fibre surface (B), as illustrated in the inset. Time course of the current pulse is shown at the top of the figure.

In most cases, the contractions of phasic type did not spread across the diameter of the fibre. In some cases, however, the phasic response spreading across the fibre diameter was observed with respect to a few sarcomeres located opposite the center of a pipette of more than $50 \mu$ diameter (Fig. 5 ).

The above-mentioned local contractions were always confined to the sarcomeres covered by the pipette, and spread along the striation pattern with a velocity of $0.7-2 \mathrm{~cm} / \mathrm{sec}$. The spreading velocity was markedly reduced by lowering temperature from 20 to $5-10^{\circ} \mathrm{C}$, giving a $\mathrm{Q}_{10}$ of $2-3$. In one case, tetrodotoxin $\left(10^{-7} \mathrm{~g} / \mathrm{ml}\right)$ was applied to a fibre showing the phasic response. The response was gradually changed from the phasic to the graded type with increasing threshold depolarization, though a time of more than 20 min was required to eliminate the phasic response.

The phasic type of local contractions observed in the present experiments may be taken to indicate that some regenerating process participates in the inward spread of activation in frog fast muscle 

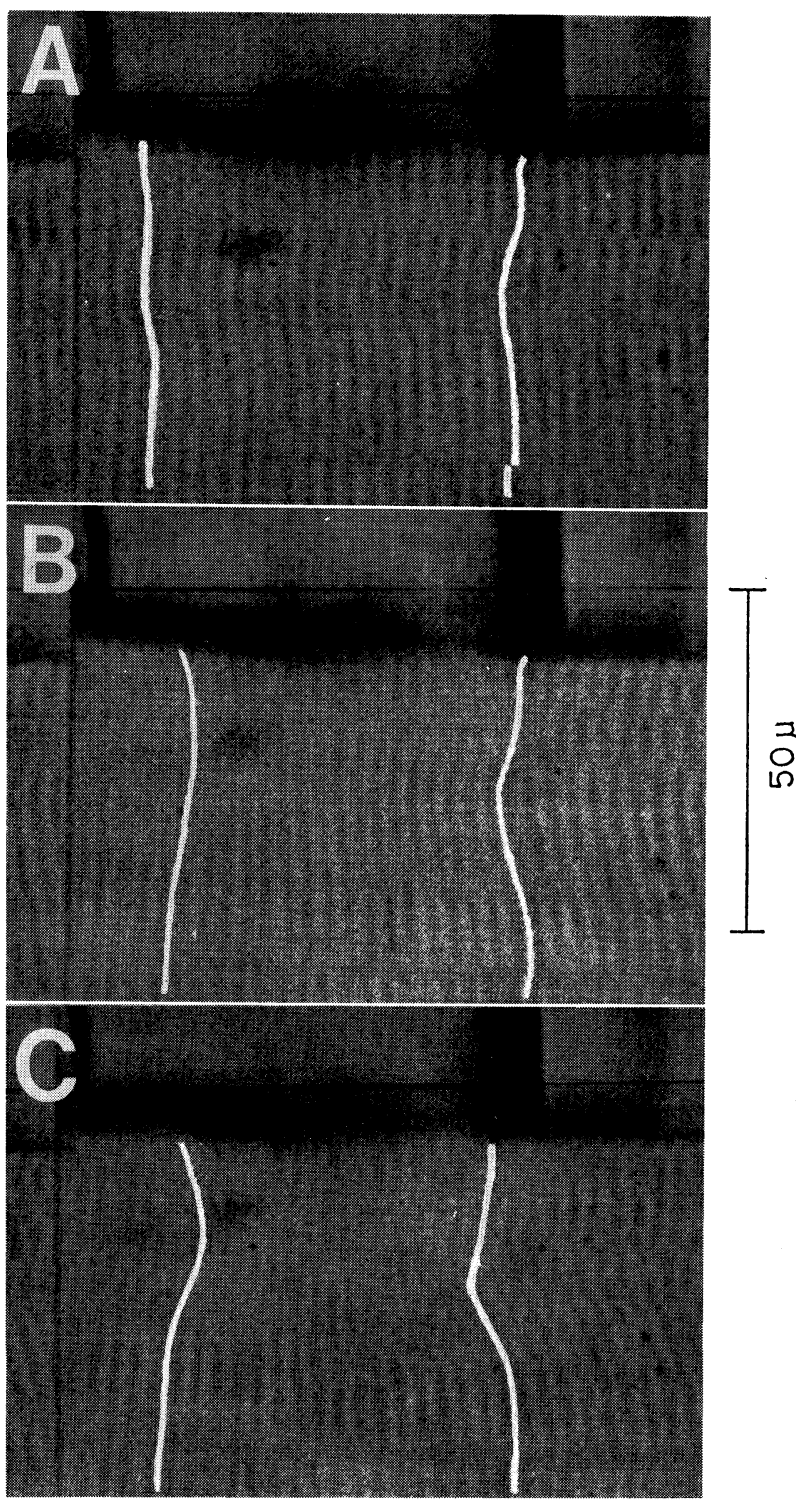

$\overbrace{\substack{0 \\ 0}}^{2}$

Fig. 3. Selected frames from a cinefilm showing a muscle fibre at rest (A) and during the local contraction of increasing extent (B and C) produced by a 500 msec current pulse producing depolarization of $20 \mathrm{mV}$. White lines in each frame mark 16 sarcomeres under the pipette. Note that the contraction is most marked at the part distant from the fibre surface where the pipette is placed. The cinerecords shown in Figs. 1 and 3 were obtained from one and the same fibre. 


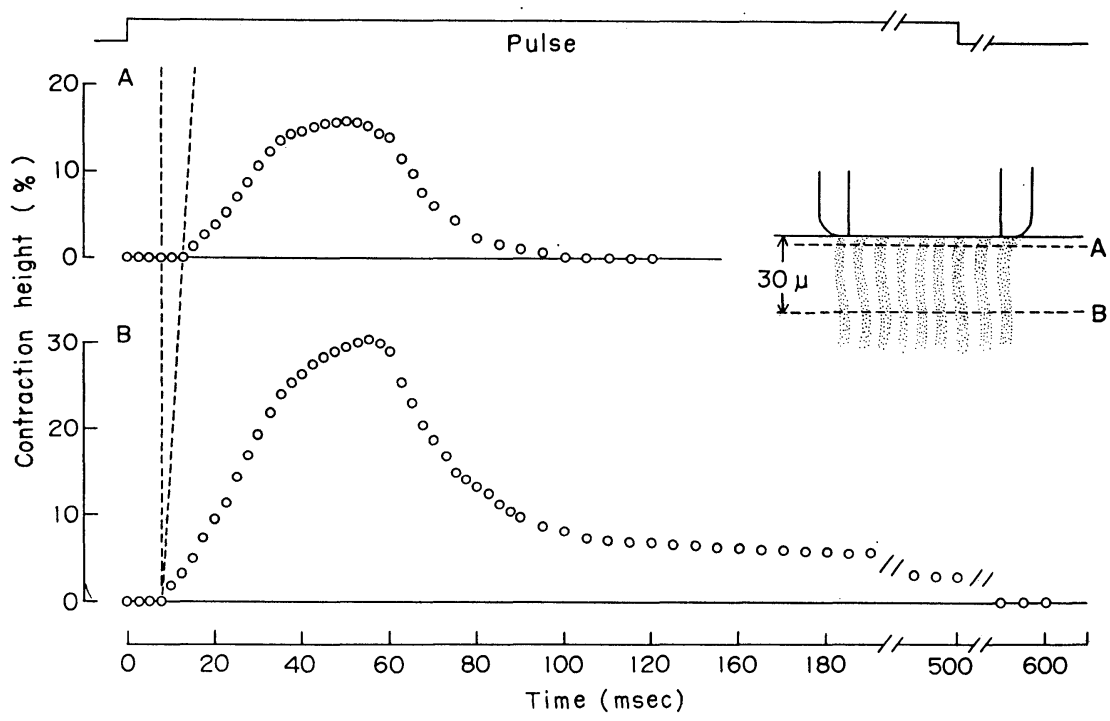

Fig. 4. Contraction curves of the local contraction shown in Fig. 3 constructed by measuring the length of 10 sarcomeres near the fibre surface (A) and at the part about $30 \mu$ distant from the fibre surface (B). Note that the contraction is first initiated at the interior of the fibre, and spreads to the fibre surface.
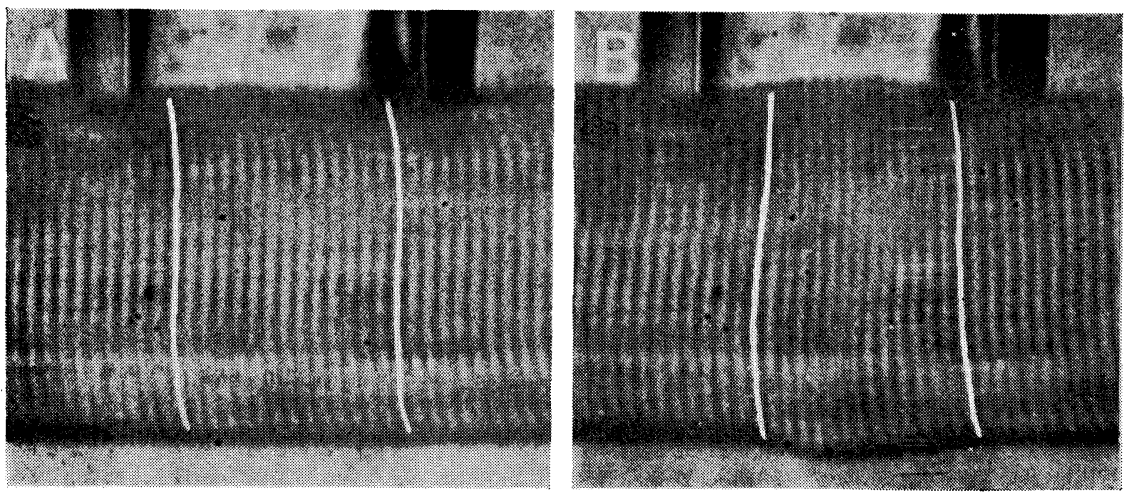

$50 \mu$

Fig. 5. Selected frames from a cinefilm of a relaxed muscle fibre (A) and of the local contraction (B) produced by a $300 \mathrm{msec}$ current pulse producing depolarization of $25 \mathrm{mV}$. White lines mark 13 sarcomeres. The contraction spreads across the fibre diameter with respect to a few sarcomeres opposite the center of the pipettet 
fibres. Another interesting finding in the present study is that, with large pipettes, the contraction could first be initiated at the interior of the fibre distant from the fibre surface, and occurred most markedly, being followed by the small steady contraction (Fig. 4). This seems to suggest that, in addition to the presence of the regenerating mechanism, the organization and the electrical properties of the $\mathrm{T}$ tubule network are such that the current density causing depolarization of the $\mathrm{T}$ tubule membrane is maximum at a part distant from the fibre surface when a sufficiently large area of the surface membrane is stimulated. Details of the present work will be published elsewhere with more experimental results.

Acknowledgement. I wish to thank Prof. K. Uchizono for his interest and encouragement. My thanks are also due to Miss M. Higuchi for her excellent technical assistance.

\section{References}

1) Benzanilla, F., Caputo, C., González-Serratos, H., and Venosa, R. A. (1972) : J. Physiol., 223, 507.

2) Costantin, L. L. (1970): J. Gen. Physiol., 55, 703.

3) González-Serratos, H. (1971) : J. Physiol., 212, 777.

4) Huxley, A. F., and Taylor, R. E. (1958): J. Physiol., 144, 426.

5) Strickholm, A. (1966): Nature, 212, 835.

6) Sugi, H., and Ochi, R. (1967): J. Gen. Physiol., 50, 2145.

7) (1967): J. Gen. Physiol., 50, 2167. 